\title{
Clinical implications of pulmonary regurgitation in healthy individuals: detection by cross sectional pulsed Doppler echocardiography
}

\author{
SEIICHI TAKAO, KUNIO MIYATAKE, SHIRO IZUMI, \\ MITSUNORI OKAMOTO, $\dagger$ NAOKAZU KINOSHITA, HIROSHI NAKAGAWA, \\ KAZUHIRO YAMAMOTO, HIROSHI SAKAKIBARA, YASUHARU NIMURA* \\ From the ${ }^{\star}$ Cardiology Division and Research Institute, National Cardiovascular Centre, Osaka, Japan, and \\ $\dagger$ First Department of Medicine, Hiroshima University, School of Medicine, Hiroshima, Japan
}

SUMmaRY Pulsed Doppler echocardiography in healthy individuals often shows a disturbance of diastolic flow in the right ventricular outflow tract just below the pulmonary valve that suggests regurgitation. This disturbance of diastolic flow was studied in 50 healthy individuals and 40 patients with cardiopulmonary disease, some of whom had a pulmonary regurgitant murmur. Diastolic flow was disturbed in 39 of the 50 healthy individuals. In 32, cross sectional echocardiography gave a satisfactory image of the pulmonary valve. The characteristic Doppler signals usually lasted throughout diastole, were directed toward the right ventricular cavity, and gradually waned towards end diastole; they formed a spindle shaped area of abnormal signals that extended to within $10 \mathrm{~mm}$ of the coaptation of the pulmonary valve towards the right ventricular cavity and the pressure difference estimated from the signals by the modified Bernoulli equation seemed to be proportional to the normal retrograde transpulmonary pressure difference. In all 40 patients with cardiopulmonary disease, signals indicating pulmonary regurgitation were found whether or not a regurgitant murmur was present. When it was present, however, the spindle was longer than $20 \mathrm{~mm}$ and in patients with pulmonary hypertension the velocity of abnormal diastolic flow was higher than in healthy individuals. The Doppler signals registering disturbed flow in the healthy individuals resembled the signals caused by pulmonary regurgitation in the patients in terms of location, orientation, and configuration.

These results show that healthy individuals usually have trivial pulmonary regurgitation. In practice the distance that the flow disturbance extends from the valve and estimated pressure difference across the valve are probably the most important variables for assessing the clinical significance of pulmonary valve regurgitation.

Doppler echocardiography is a sensitive and specific technique for detecting valve regurgitation. ${ }^{1-4}$ Even in healthy subjects, unusual Doppler signals are often seen just beneath the pulmonary valve in the right ventricular outflow tract during diastole; these are apparently signals from pulmonary regurgitation. We have examined a series of healthy individuals for these signals to find out whether they indicate true regurgitation and are clinically significant.

Requests for reprints to Dr Kunio Miyatake, Cardiology Division, National Cardiovascular Center, 7-1, Fujishiro-dai 5-chome, Suita, Osaka 565, Japan.

Accepted for publication 8 September 1987

\section{Patients and methods}

We examined 50 healthy individuals aged 23 to 59 years (mean 35 years) and 40 patients with cardiopulmonary disease aged 19 to 68 years (mean 40 years). The 50 healthy individuals had no history of cardiopulmonary disease and no abnormality on physical examination and cross sectional echocardiography. The $\mathbf{4 0}$ patients with cardiopulmonary disease all had echocardiograms that clearly showed the right ventricular outflow tract and pulmonary valve. Nineteen had acquired valve disease, 14 had congenital heart disease, two had cardiomyopathy, two had idiopathic dilatation of the pulmonary 
artery, two had pulmonary embolism, and one had primary pulmonary hypertension. Phonocardiography performed simultaneously with the Doppler examination showed a pulmonary regurgitant murmur in 12 of the 40 patients. The remaining 28 patients did not have a diastolic murmur.

\section{METHODS}

\section{Equipment}

We used two commercially available cross sectional pulsed Doppler echocardiograms, a Toshiba SSH11A/SDS-10A and a SSH-40A/SDS-21A. The transducer frequency was $2.4 \mathrm{MHz}$ and the pulse repetition rate was 6000 bursts/s $(1 \mathrm{kHz}$ of Doppler frequency is equivalent to $31 \mathrm{~cm} / \mathrm{s}$ flow velocity). The sample volume size was $4 \times 4 \mathrm{~mm}$ in width and $2 \mathrm{~mm}$ in depth. A $400 \mathrm{~Hz}$ high-pass filter was used to eliminate artefacts caused by cardiac wall motion. The frequency analysis was carried out by fast Fourier transformation. Doppler beam direction and the site of sample volume were displayed on the cross sectional echo image of the heart. Analysed Doppler signals were displayed as a sound spectrogram with the electrocardiogram, phonocardiogram, and $M$ mode echocardiogram on a strip chart recording paper (paper speed $50 \mathrm{~mm} / \mathrm{s}$ ). Flow components towards and away from the transducer were displayed as flow signals above and below the baseline, respectively.

Ten of the 50 healthy individuals were also examined by colour-coded real time cross sectional Doppler flow imaging to confirm the finding obtained by the conventional method. ${ }^{5}$ In this technique, Doppler flow signals were processed by the high-speed autocorrelation technique, so that flow direction, mean velocity, and degree of flow dispersion were simultaneously displayed on the same site in the cross sectional echo image of the heart with colour codes in real time. A red code indicated the flow component toward the transducer and blue showed flow away from the transducer. The colour intensity expressed the magnitude of flow velocity and green was added to the red or blue to show the degree of flow dispersion.

\section{SEARCHING TECHNIQUE}

The sagittal cross sectional view of the right ventricular outflow tract including the pulmonary valve was obtained through the third or fourth intercostal space. Then the transducer was gradually tilted to obtain sagittal cross sectional views over the whole right ventricular outflow tract. We analysed the flow pattern on each cross sectional plane. The area just beneath the pulmonary valve was examined for jets in diastole. In the healthy individuals Doppler signals indicating jet flow were detected near the pulmonary valve in the right ventricular outflow tract during diastole. These signals had a wide velocity spectrum with flow away from the pulmonary valve and lasted more than $200 \mathrm{~ms}$ in diastole (fig 1a). In early diastole signals lasting $<200 \mathrm{~ms}$ were not considered because in that phase it is difficult to exclude the influence of the valve and wall motion on the record.

When Doppler signals indicating disturbed diastolic flow were detected we recorded the cross sectional cut that showed the full extent of the signal. This view was frozen in mid diastole and the distribution of the signal was mapped by gradually changing the beam direction as described elsewhere. ${ }^{67}$

Quality of the echocardiographic image of the pulmonary valve

The quality of the echocardiographic image of the pulmonary valve was graded as: good (visualisation of two or three cusps of the pulmonary valve); poor (only one cusp visualised); none (none of the cusps was clearly visualised).

\section{Results}

FREQUENCY OF ABNORMAL DOPPLER SIGNALS IN HEALTHY INDIVIDUALS

Doppler signals indicating a jet-like disturbance of flow were detected in $39(78 \%)$ of the 50 healthy individuals examined. The quality of the echo image of the pulmonary valve was good in 32 and poor in 11 . In the remaining seven, the pulmonary valve was not visualised. Doppler signals showing a jet-like flow disturbance were detected in all 32 healthy individuals with the good image of the pulmonary valve, in seven of the 11 subjects with the poor image, and in none of the seven subjects whose valves were not visualised (table). Thus the disturbance of diastolic flow was invariably detected when the quality of the echo image of the pulmonary valve was good.

CHARACTERISTIC FEATURES OF SIGNALS SHOWING JET-LIKE FLOW DISTURBANCE IN DIASTOLE

In diastole disturbed Doppler signals were found in a small spindle-shaped region extending from the coaptation site of the pulmonary valve into the right

Table Frequency of disturbed Doppler signals showing a jetlike flow in the subpulmonary region in relation to the quality of the echocardiographic image of the pulmonary value

\begin{tabular}{llll}
\hline Image quality & Detected & Not detected & Total \\
\hline Good & 32 & 0 & 32 \\
Poor & 7 & 4 & 11 \\
None & 0 & 7 & 7 \\
\hline
\end{tabular}



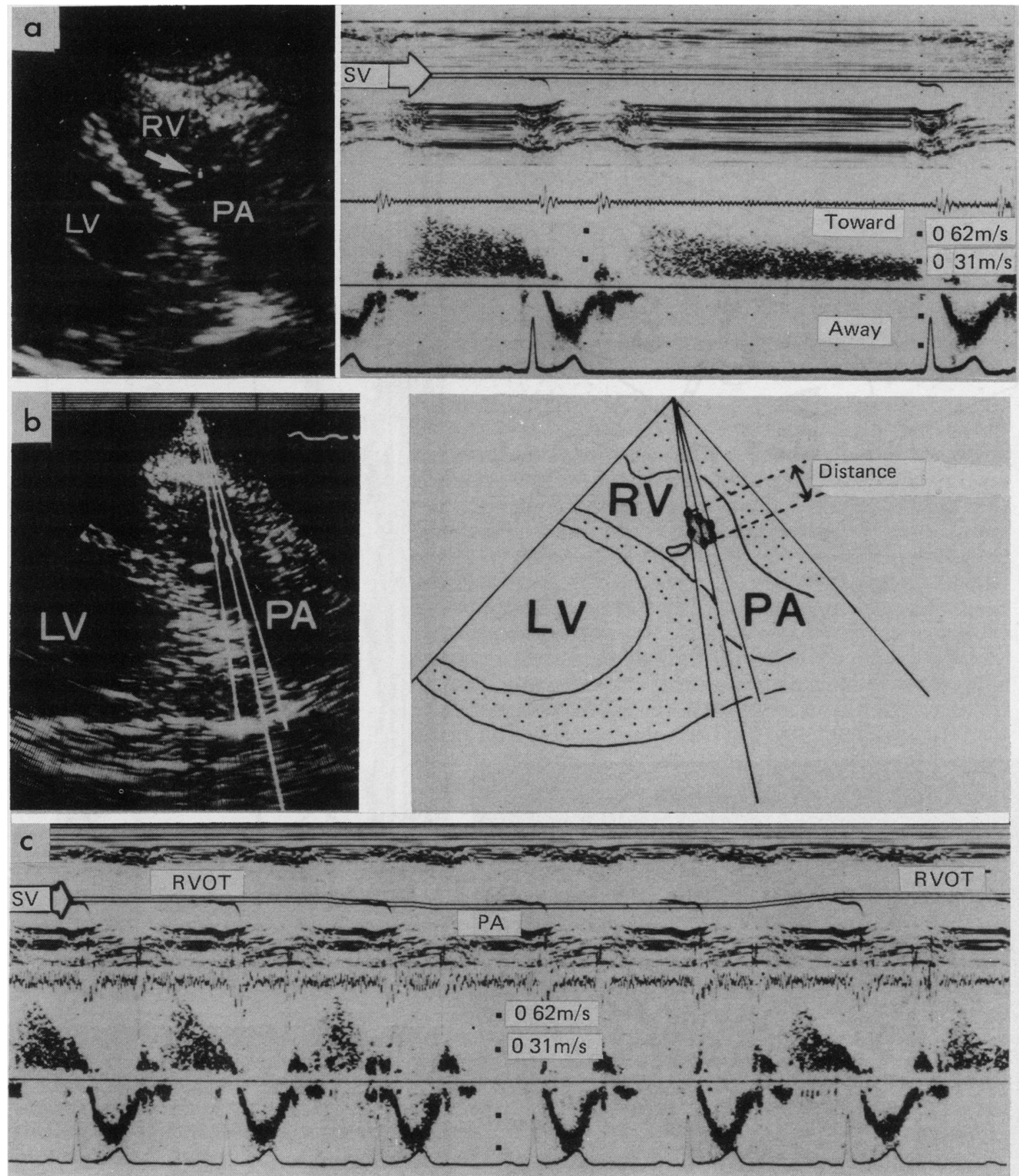

Fig 1 Doppler signals showing jet-like disturbance of flow just beneath the pulmonary valve (a) Left: Parasternal sagittal view of the basal part of the heart. The broken line indicates the direction of the Doppler beam, and the sample volume is represented by a large dot (arrow). The sample volume lay just beneath the coaptation site of the pulmonary valve in the right ventricular outflow tract. Right: Doppler signals recorded in diastole at the sample volume indicated in (a) left. The signals show a wide-band velocity spectrum away from the pulmonary valve. The flow velocity gradually decreased toward end diastole from the maximum of about $0.7 \mathrm{~m} / \mathrm{s}$ in early diastole. (b) Left: parasternal sagittal view. The subpulmonary area was searched for the signals of flow disturbance in diastole (see the change in the beam direction and the site of sample volume on each beam). The positions of the thick white dots show where such signals were detected. (b) Right: diagrammatic representation of (b) left. The maximum distance reached by the signals of flow disturbance is shown. (c) M mode echocardiogram of the pulmonary area and the frequency spectrogram of the signals of flow disturbance obtained there. The location of the sample volume in diastole was gradually shifted from near the pulmonary valve in the right ventricular outflow tract to the pulmonary trunk and then back again. Signals showing disturbed flow in diastole appeared only near the pulmonary valve and never in the pulmonary trunk. $R V$, right ventricle; $L V$, left ventricle; $P A$, pulmonary artery; $R V O T$, right ventricular outflow tract; $S V$, sample volume. 

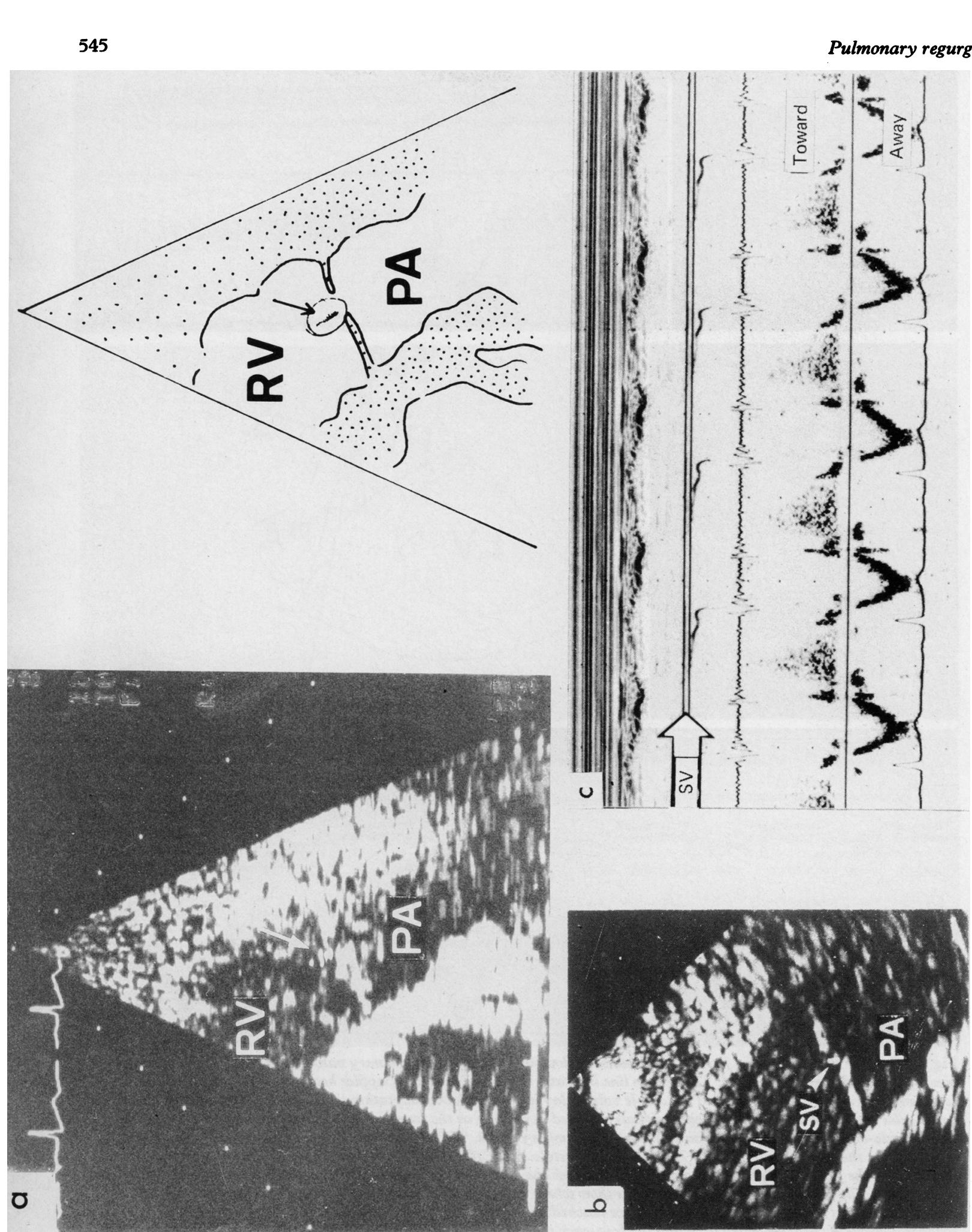

gitation

$\underline{\underline{\sigma}}$

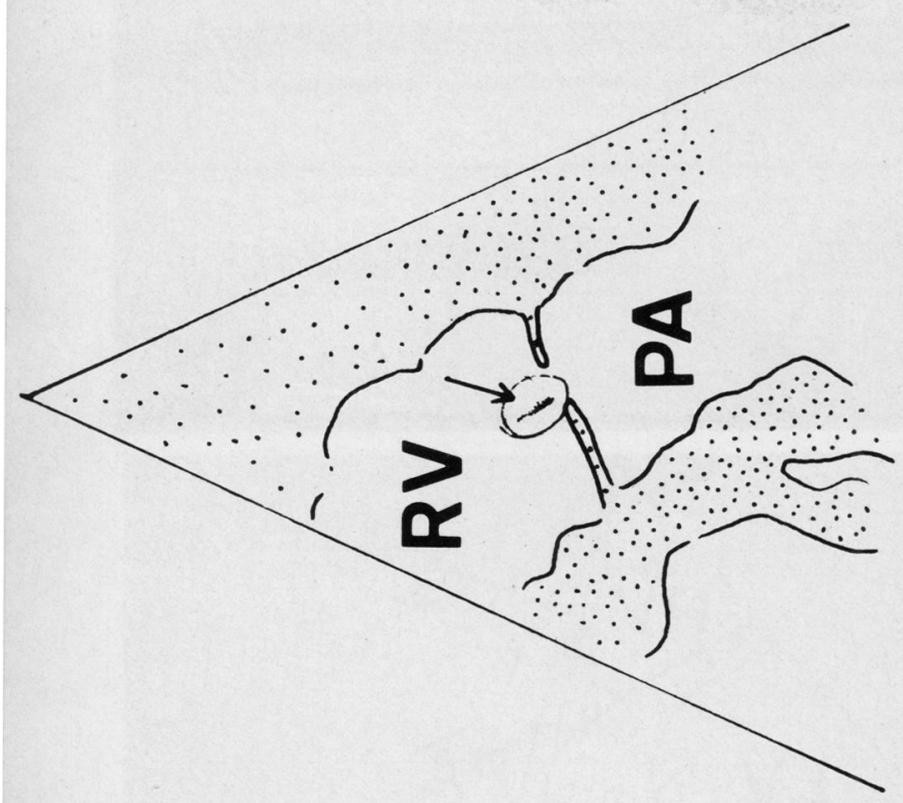


ventricular outtlow tract, but they were never found in the pulmonary trunk (fig 1 ). They were audible as high pitched decrescendoes that resembled signals caused by aortic and pulmonary regurgitation. Although none of the healthy subjects had a murmur indicating pulmonary regurgitation, the unusual Doppler signals were almost indistinguishable from the pulmonary regurgitant signals obtained from patients with pulmonary regurgitant murmurs in terms of flow direction, distribution, and other properties.

The maximal distances to which the signals of the flow disturbance extended from the pulmonary orifice were $\leqslant 10 \mathrm{~mm}$ except in one case in which the distance was $12 \mathrm{~mm}$ (fig $1 \mathrm{~b}$ ). The velocity of these Doppler signals was fastest in early diastole and then gradually declined towards end diastole (fig 1a). The maximal velocity of the diastolic disturbed flow in one pulse period ranged from 0.7 to $1.5 \mathrm{~m} / \mathrm{s}$ in healthy subjects. According to the modified Bernoulli equation, this velocity corresponds to pressure difference of about 2 to $9 \mathrm{~mm} \mathrm{Hg}$.

The real time cross sectional Doppler flow imaging technique was applied to ten of the 39 healthy subjects in whom Doppler signals, showing a jet-like flow disturbance, were detected by the conventional method. It invariably showed a trivial, but definite, jet flow spurting from the coaptation site of the pulmonary valve into the right ventricular cavity (fig 2). The characteristics of these Doppler signals were identical with those seen in the patients with pulmonary regurgitation.

DISTINGUISHING THE JET-LIKE DISTURBED FLOW FROM OTHER FLOWS IN THE RIGHT VENTRICULAR OUTFLOW TRACT

Flow velocity measured around the jet-like disturbed flow fell off abruptly. No other rapid flows were detected near the jet-like flow in the right ventricular outflow tract during diastole. Careful examination, however, showed two kinds of slow stream near the pulmonary valve in the right ventricular outflow tract in diastole (fig 3). One was a flow toward the pulmonary valve in the anterior and central parts of the outflow tract, and the other was a flow away from the pulmonary valve, on the septal side. The flow towards the valve seemed to be the tricuspid inflow reaching the outflow tract. Reversal of this flow just beneath the pulmonary valve may give rise to the flow away from the valve. The jet-like disturbed flow was quite different from these flows in velocity and topography.
SIMILARITY BETWEEN JET-LIKE FLOW DISTURBANCE SIGNALS IN HEALTHY SUBJECTS AND REGURGITANT SIGNALS IN PATIENTS WITH PULMONARY REGURGITATION

Twelve of the 40 patients with cardiopulmonary disease had a pulmonary regurgitant murmur. This produced jet-like signals from the coaptation site into the right ventricular cavity. Eight of these 12 patients had pulmonary hypertension. The frequency spectrogram showed multiple aliasing, indicating that the velocity was higher than the upper limit of the measurable range (that is, $2 \mathrm{~m} / \mathrm{s}$ ) and the upper limit of the healthy subjects $(1.5 \mathrm{~m} / \mathrm{s})$. In the remaining four patients (two with idiopathic dilatation of the pulmonary artery and two with tetralogy of Fallot after operation) the flow velocity was within the normal range. The distance reached by the regurgitant flow was $20 \mathrm{~mm}$ or further in all 12 patients (fig 4).

In the 28 patients without a pulmonary regurgitant murmur, we detected jet-like Doppler signals from the coaptation site of the pulmonary valve. Although these signals resembled the pulmonary regurgitant signals in configuration and orientation, they were generally shorter ( $>20 \mathrm{~mm}$ in two patients, between 10 and $20 \mathrm{~mm}$ in 16 , and $<10 \mathrm{~mm}$ in 10). Although in an earlier study we reported that these signals indicate silent pulmonary regurgitation, ${ }^{4}$ in the last 10 patients the signals $(<10 \mathrm{~mm})$ were identical to those of the jet-like flow disturbance that we found in healthy individuals.

\section{Discussion}

DO THE DOPPLER SIGNALS SHOWING A JET-LIKE DISTURBANCE OF FLOW IN THE RIGHT VENTRICULAR OUTFLOW TRACT OF HEALTHY SUBJECTS INDICATE TRUE PULMONARY REGURGITATION?

With the pulsed Doppler technique, valve regurgitation is diagnosed when high-frequency Doppler signals with wide-band spectrum are detected extending backward from the malfunctioning valve. ${ }^{7-9} \mathrm{We}$ found Doppler signals, showing a jet-like disturbance of flow in healthy subjects, with the following features: (1) a long duration in diastole; (2) spurting from the coaptation site of the pulmonary valve, with velocity toward the right ventricular cavity; (3) spindle-like configuration; (4) and a flow velocity that was nearly the same as the transpulmonary pressure difference. The falling off in velocity towards end diastole also corresponds to the time course of the transpulmonary pressure gradient in diastole. Apart from the distance reached by the jet all features resemble the signals recorded in pulmonary regurgitation. In those patients with cardiopulmonary 

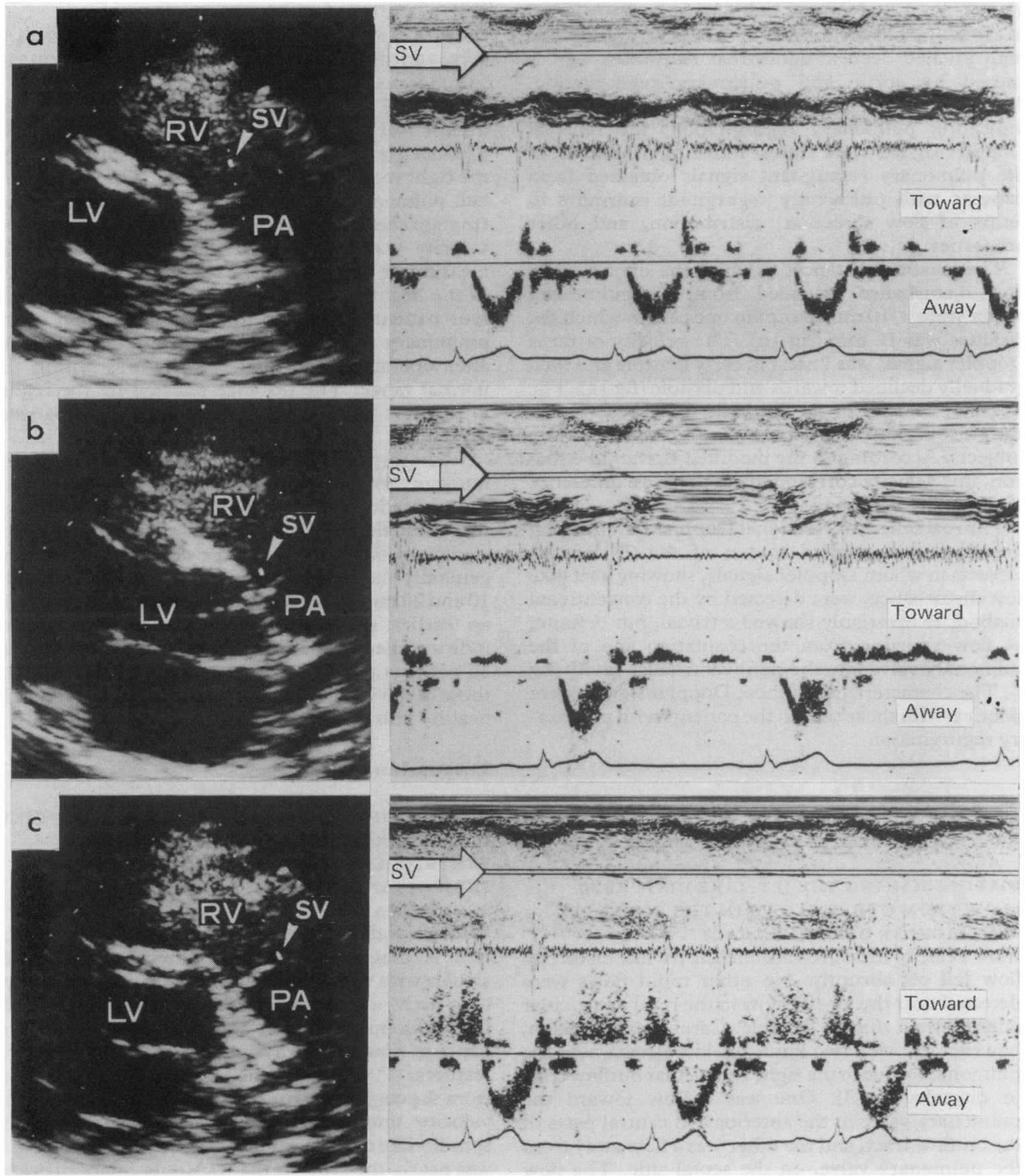

Fig 3 Doppler signals surrounding the jet-like disturbance of flow in the subpulmonary region in a healthy 28 year old man. (a) Left: sample volume for Doppler signals lies near the pulmonary valve in the central part of the right ventricular outflow tract (arrow). (a) Right: slow flow towards the pulmonary valve was recorded by the sample volume (a, left) in diastole.

(b) Slow flow away from the pulmonary valve was recorded near the interventricular septum. (c) There were Doppler signals with a wide-band frequency spectrum in a localised area close to the coaptation site of the pulmonary valve. These were distinct from the flows seen in $a$ and $b$. See fig 1 for abbreviations. 

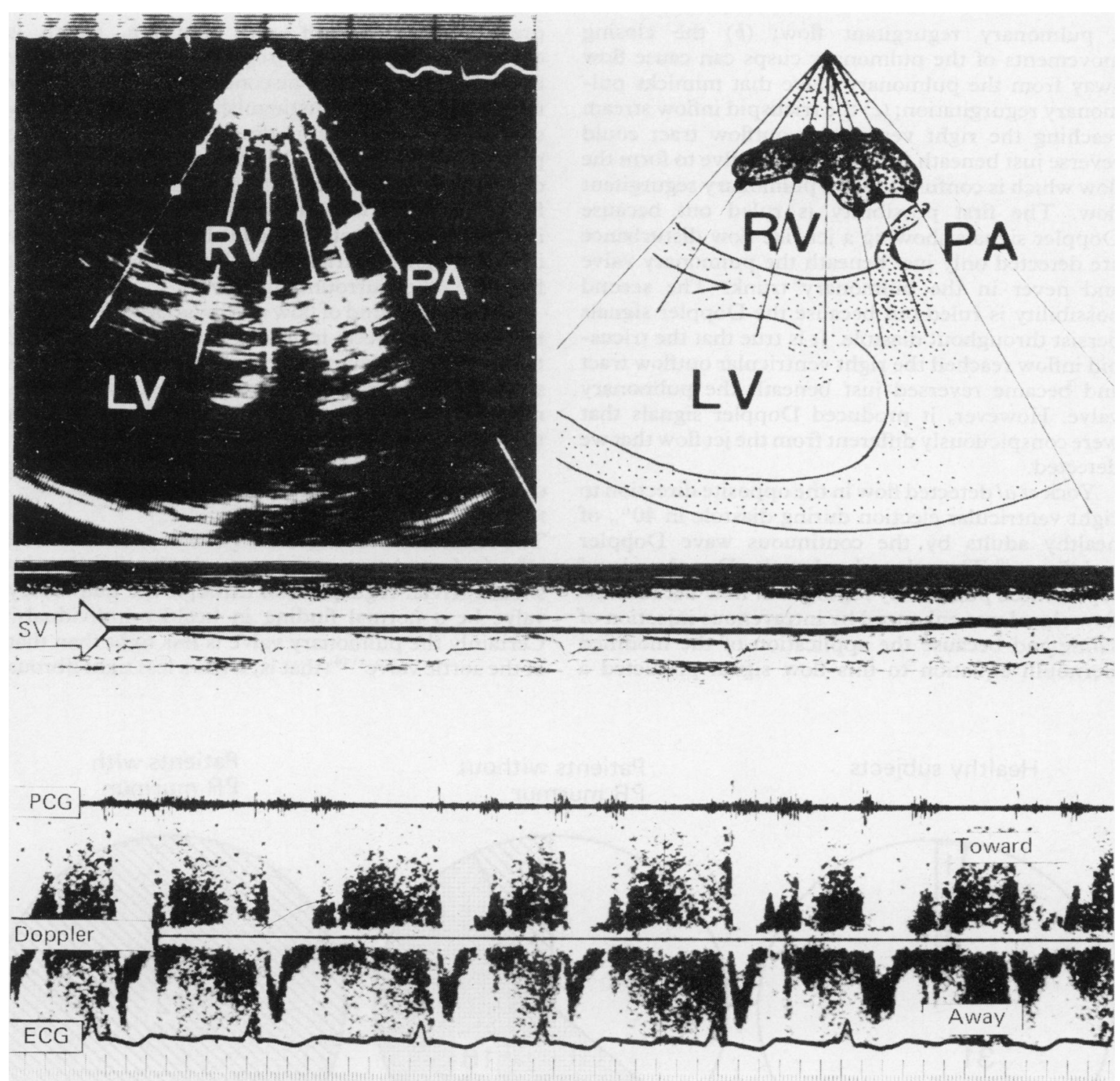

Fig 4 Doppler signals in a 40 year old woman with Eisenmenger's syndrome and a pulmonary regurgitant murmur.

Parasternal sagittal view of the basal part of the heart and its diagram (above). The region where pulmonary regurgitant loppler signals were detected is shown by thick white dots. The frequency spectrogram of the pulmonary regurgitant Doppler signals (below) shows aliasing, indicating that the regurgitant jet had a high velocity caused by pulmonary hypertension. See fig 1 for abbreviations.

disease who might have silent pulmonary regurgitation, the length of the jet was less than that in the patients with documented pulmonary regurgitation and more than that in the healthy subjects. This continuous spectrum of Doppler signals from confirmed pulmonary regurgitation to normality suggests that when a jet-like flow disturbance is found in healthy subjects it should be interpreted as trivia pulmonary regurgitation.

But there may be explanations other than regurgitation through the pulmonary valve for this flow disturbance in healthy subjects: $(a)$ the pulmonary ejection flow which reverses just above the pulmonary valve in the pulmonary trunk may be mistaken for 
a pulmonary regurgitant flow; (b) the closing movements of the pulmonary cusps can cause flow away from the pulmonary valve that mimicks pulmonary regurgitation; (c) the tricuspid inflow stream reaching the right ventricular outflow tract could reverse just beneath the pulmonary valve to form the flow which is confused with a pulmonary regurgitant flow. The first possibility is ruled out because Doppler signals showing a jet-like flow disturbance are detected only just beneath the pulmonary valve and never in the pulmonary trunk. The second possibility is ruled out because the Doppler signals persist throughout diastole. It is true that the tricuspid inflow reached the right ventricular outflow tract and became reversed just beneath the pulmonary valve. However, it produced Doppler signals that were conspicuously different from the jet flow that we detected.

Yock et al detected flow in the opposite direction to right ventricular ejection during diastole in $40^{\circ}{ }^{\circ}$ of healthy adults by the continuous wave Doppler technique. ${ }^{10}$ They thought that the Doppler signal represented pulmonary regurgitant flow because the flow signal was enhanced by intravenous injection of saline and because the application of the modified Bernoulli equation to this flow signal predicted a pressure difference of $<10 \mathrm{~mm} \mathrm{Hg}$, which is equivalent to the retrograde transpulmonary difference in diastole. The continuous wave Doppler technique does not distinguish the abnormal flow signals from the surrounding flow signals. In the present study, the pulsed Doppler technique clearly demonstrated that the Doppler signals from jet-like flow disturbance were confined to the region extending from the coaptation site of the pulmonary valve into the right ventricular cavity and were distinct from the other surrounding flows.

Because this kind of flow was invariably detected in the healthy subjects in whom the coaptation site of the pulmonary valves was clearly imaged on the cross sectional echocardiogram, we assume that it is normal for healthy subjects to have trivial regurgitation through the pulmonary valve.

\section{CAUSES OF REGURGITATION THROUGH THE NORMAL PULMONARY VALVE}

The semilunar valves are designed to eject the blood effectively and to prevent regurgitation. ${ }^{11}{ }^{12}$ So why should trivial regurgitation through the pulmonary valve be a normal finding in healthy individuals? Certainly the pulmonary valve is less tight than that of the aortic valve $\mathrm{1}^{1314}$ (that is, it has a less tight fibrous
Healthy subjects

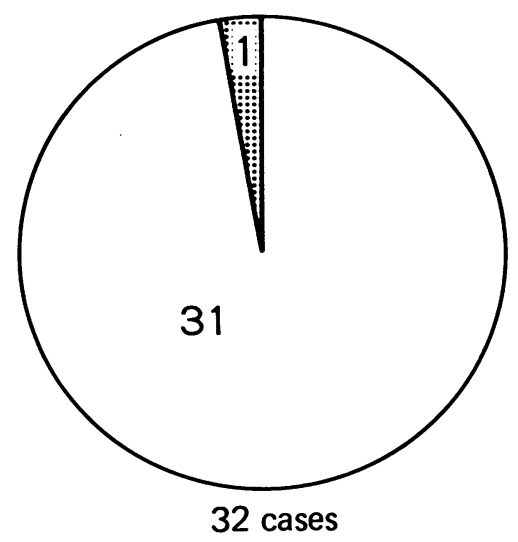

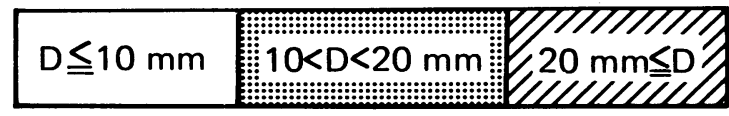

Patients without PR murmur

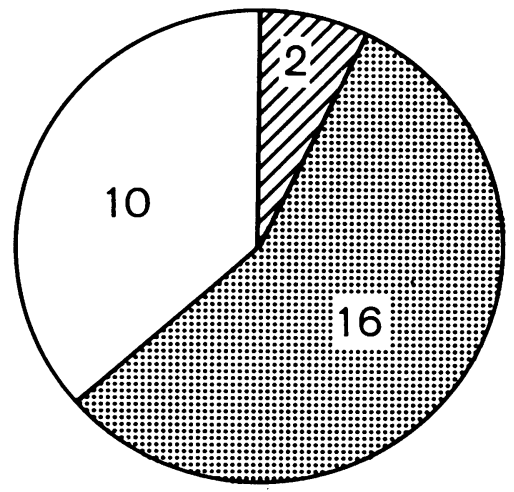

28 cases

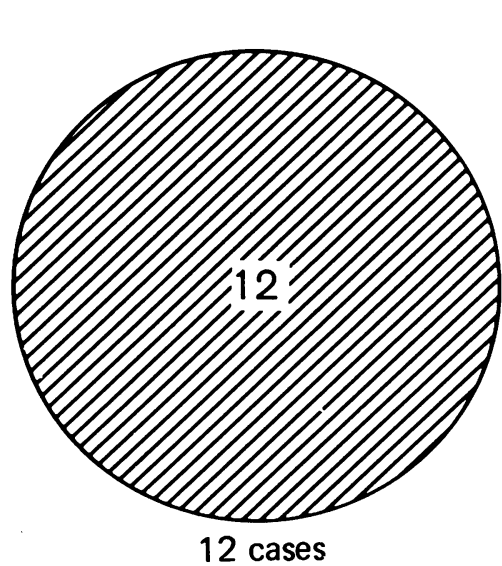

12 cases

Patients with

PR murmur

Fig 5 The maximum distances reached by the diastolic disturbed flow signal in healthy subjects and in patients with cardiopulmonary disease. The maximum distance was measured in 32 healthy subjects in whom the pulmonary valve was well visualised. The distance was $\leqslant 10 \mathrm{~mm}$ in 31 of them. In all the patients with a pulmonary regurgitant murmur the distance was $\geqslant 20 \mathrm{~mm}$. In the group of patients with no pulmonary regurgitant murmur the maximum distance lay between that of the healthy group and the group with a murmur. $D$, maximum distance of regurgitant signal; $P R$, pulmonary regurgitant. 
ring, thinner cusps, a more hypoplastic Alantius nodule, and shallower sinuses). Furthermore, the retrograde pressure gradient through the pulmonary valve in diastole may not be enough to close the valve as tightly as the aortic valve.

This tendency to regurgitation may mitigate against lung congestion by moderating the pressure rise in the pulmonary capillaries by reducing the excessive preload to the left side of the heart.

\section{CLINICAL IMPLICATION}

Patients with rheumatic heart disease often have Doppler signals typical of pulmonary regurgitation. ${ }^{34}$ In some, these were thought to indicate silent pulmonary regurgitation. ${ }^{4}$ The present study, however, shows that such signals are not always abnormal, because healthy individuals commonly have trivial pulmonary regurgitation. Therefore, whenever pulmonary regurgitant signals are detected, their clinical significance should be assessed before they are attributed to cardiopulmonary diseases.

The signals have two important properties. The first is velocity, which may reflect a retrograde transpulmonary pressure difference during diastole. In healthy individuals the transpulmonary pressure gradient is small in diastole. Maximal velocity was $<1.5 \mathrm{~m} / \mathrm{s}$ of all our healthy individuals, and this is consistent with the normal retrograde transpulmonary pressure difference expected of $<4 \times 1.5^{2}=9$ $\mathrm{mm} \mathrm{Hg}$ (modified Bernoulli equation). In those with pulmonary hypertension, the maximal velocity of the pulmonary regurgitation was well above the physiological range. In patients with pulmonary regurgitant murmur, but not pulmonary hypertension, the regurgitant flow velocity was the same as that in healthy subjects. This finding is discussed below.

The second important property of the pulmonary regurgitant signal is the maximum distance travelled by the signals from the pulmonary orifice into the right ventricle. The maximum distance travelled by regurgitant signals from the regurgitant orifice correlated roughly with the degree of regurgitation. ${ }^{67}$ While the distance was usually $\leqslant 10 \mathrm{~mm}$ in the healthy individuals, it was $\geqslant 20 \mathrm{~mm}$ in all 12 patients with pulmonary regurgitant murmurs in whom regurgitation was an expected finding. Distances of $\leqslant 10 \mathrm{~mm}$ may be accepted as being within the physiological range, those $>10 \mathrm{~mm}$ to $20 \mathrm{~mm}$ as borderline, and those $\geqslant 20 \mathrm{~mm}$ as indicating clinically important regurgitation (fig 5).

The figure of $10 \mathrm{~mm}$ is not an absolute marker for pulmonary regurgitation. This is because it is difficult to measure accurately the precise distance reached by the pulmonary regurgitant signal because the right ventricular outflow tract is anatomically complex. There are still problems with resolution and gain with current equipment. Nevertheless, these difficulties should not obscure the importance of measuring this distance which, together with the velocity, is the major determinant for assessing the clinical significance of signals indicating pulmonary regurgitation.

This work was supported in part by the Grant-in Aid for Development Scientific Research from the Ministry of Education, Science and Culture of Japan, 1984, 1985.

\section{References}

1 Ward JM, Baker DW, Rubenstein SA, Johson SL. Detection of aortic insufficiency by pulse Doppler echocardiography. JCU 1977;1:5-10.

2 Richards KL, Cannon SR, Crawford MH, Sorensen SG. Noninvasive diagnosis of aortic and mitral valve disease with pulsed-Doppler spectral analysis. $\mathrm{Am} \mathrm{J}$ Cardiol 1983;51:1122-7.

3 Waggoner AP, Quinones RMA, Young JB, et al. Pulsed Doppler echocardiographic detection of right-sided valve regurgitation. Am J Cardiol 1981;47:279-86.

4 Miyatake K, Okamoto M, Kinoshita N, et al. Pulmonary regurgitation studied with the ultrasonic pulsed Doppler technique. Circulation 1982;65:969-76.

5 Miyatake $\mathrm{K}$, Okamoto $\mathrm{M}$, Kinoshita $\mathrm{N}$, et al. Clinical applications of a new type of real-time twodimensional Doppler flow imaging system. $A m J$ Cardiol 1984;54:857-68.

6 Miyatake K, Kinoshita N, Nagata S, et al. Intracardiac flow pattern in mitral regurgitation studied with combined use of the ultrasonic pulsed Doppler technique and cross-sectional echocardiography. $\mathrm{Am} \mathrm{J}$ Cardiol 1980;45:155-62.

7 Miyatake K, Okamoto M, Kinoshita N, et al. Evaluation of tricuspid regurgitation by pulsed Doppler and two-dimensional echocardiography. Circulation 1982;66:777-84.

8 Stevenson JG, Kawabori I, Guntheroth WG. Differentiation of ventricular septal defects from mitral regurgitation by pulsed Doppler echocardiography. Circulation 1977;56:14-8.

9 Baker DW. Rubenstein SA, Lorch GS. Pulsed Doppler echocardiography: principle and applications. Am J Cardiol 1977;63:69-80.

10 Yock PG, Naasz C, Schnittger I, Popp RL. Doppler tricuspid and pulmonic regurgitation in normals: is it real? [Abstract] Circulation 1984;70 (suppl II):II-40.

11 Thubrikar M, Harry R, Nolan SP. Normal aortic valve function in dogs. Am J Cardiol 1977;40:563-8.

12 Thubrikar M, Piepgrass WC, Shaner TW, Nolan SP. The design of the normal aortic valve. Am J Physical 1981;24:H795-801.

13 Gross L, Kugel MA. Topographic anatomy and histology of the valves in the human heart. Am J Pathol 1931;7:445-73.

14 Silver MM. Gross examination and structure of the heart. In: Silver MD, ed. Cardiovascular pathology. New York, Edinburgh, London, Melbourne: Churchill Livingstone, 1983:13. 